\title{
IceCube Search for Earth-traversing ultra-high energy Neutrinos
}

\section{The IceCube Collaboration}

(a complete list of authors can be found at the end of the proceedings)

E-mail: isafa@fas.harvard.edu

The search for ultra-high energy neutrinos is more than half a century old. While the hunt for these neutrinos has led to major leaps in neutrino physics, including the detection of astrophysical neutrinos, neutrinos at the $\mathrm{EeV}$ energy scale remain undetected. Proposed strategies for the future have mostly been focused on direct detection of the first neutrino interaction, or the decay shower of the resulting charged particle. Here we present an analysis that uses, for the first time, an indirect detection strategy for EeV neutrinos. We focus on tau neutrinos that have traversed Earth, and show that they reach the IceCube detector, unabsorbed, at energies greater than $100 \mathrm{TeV}$ for most trajectories. This opens up the search for ultra-high energy neutrinos to the entire sky. We use ten years of IceCube data to perform an analysis that looks for secondary neutrinos in the northern sky, and highlight the promise such a strategy can have in the next generation of experiments when combined with direct detection techniques.

Corresponding authors: Ibrahim Safa ${ }^{1,2, *}$, Carlos Argüelles ${ }^{1}$

${ }^{1}$ Department of Physics and Laboratory for Particle Physics and Cosmology, Harvard University Cambridge, MA 02138, USA

${ }^{2}$ Dept. of Physics and Wisconsin IceCube Particle Astrophysics Center, University of Wisconsin-Madison Madison, WI 53706, USA

${ }^{*}$ Presenter

$37^{\text {th }}$ International Cosmic Ray Conference (ICRC 2021)

July 12th-23rd, 2021

Online - Berlin, Germany 


\section{Introduction}

The IceCube Neutrino Observatory is a gigaton-scale unsegmented ice-Cherenkov detector located at the geographical South Pole. Since its completion in 2012, IceCube has measured an extraterrestrial high-energy diffuse neutrino flux, whose sources are yet largely unidentified. The observed astrophysical flux has been measured between $\mathrm{O}(10 \mathrm{TeV})$ and $\mathrm{O}(1 \mathrm{PeV})$ and is welldescribed by an unbroken power-law [1-3]. IceCube has also reported the first hints of astrophysical neutrino sources in 2017, with the observation of neutrinos from the blazar TXS 0506+056 [4, 5]. More recently, analyses with more years of data and updated tools and reconstructions revealed NGC 1068 as a possible neutrino source [6].

Despite its success, IceCube has not observed neutrinos produced by interactions of cosmicrays with cosmic microwave background (CMB) light. This cosmogenic neutrino flux, also known as the GZK flux due to the expected associated cut-off in the cosmic-ray spectrum, is a major target of current and next-generation neutrino telescopes. Moreover, predictions of neutrino fluxes from certain populations such as FSRQs and high-luminosity AGN jets show peaks around an EeV in energy with little emission at $\mathrm{TeV}-\mathrm{PeV}$, suggesting some sources may only be resolved at the highest energies [7, 8]. Searches for ultra-high energy neutrinos have been performed by looking at through-going very-high energy events in IceCube [9] and by looking at Earth-skimming events in cosmic-ray detectors such as the Pierre Auger Observatory, and with radio detectors such as ANITA, ARA, and ARIANNA [10-20]. In this contribution, we present the application of a new method to detect GZK neutrinos, which was first introduced in [21], and estimate the sensitivity of a search carried out with currently available IceCube data.

This new method relies on observing secondary neutrinos produced as tau neutrinos with $\mathrm{EeV}$ energies traverse the Earth. Secondary tau neutrinos produced in the cascading down process accumulate at energies close to $1 \mathrm{PeV}$ for most trajectories through Earth, where the current astrophysical measurements have been performed. This accumulation implies that the angular and energy distribution of the UHE component is different from that of astrophysical neutrinos. A schematic illustrating signatures from such neutrinos is shown in Fig. 1. In the following we will describe an analysis to search for these neutrinos, and provide preliminary sensitivities to some benchmark ultra-high energy neutrino models.

\section{Analysis description}

We use the event selection described in [22], which selected for northern-sky through-going muons and achieves plateauing efficiency above a few $\mathrm{TeV}$ s in energy. We use the LeptonInjector Monte Carlo [23] to generate through-going muons, which could be produced either by muon-neutrino charged-current interactions or by tau-neutrino interactions where the tau decays leptonically to a muon; the latter process happens in $18 \%$ of the tau decays. Given that LeptonInjector does not simulate neutrinos, but charged particles (or final states) that can be matched aposteriori to several physical processes, we can add the contribution of the tau-neutrino interactions to muon tracks via reweighting. The effective tau weight is given by, 


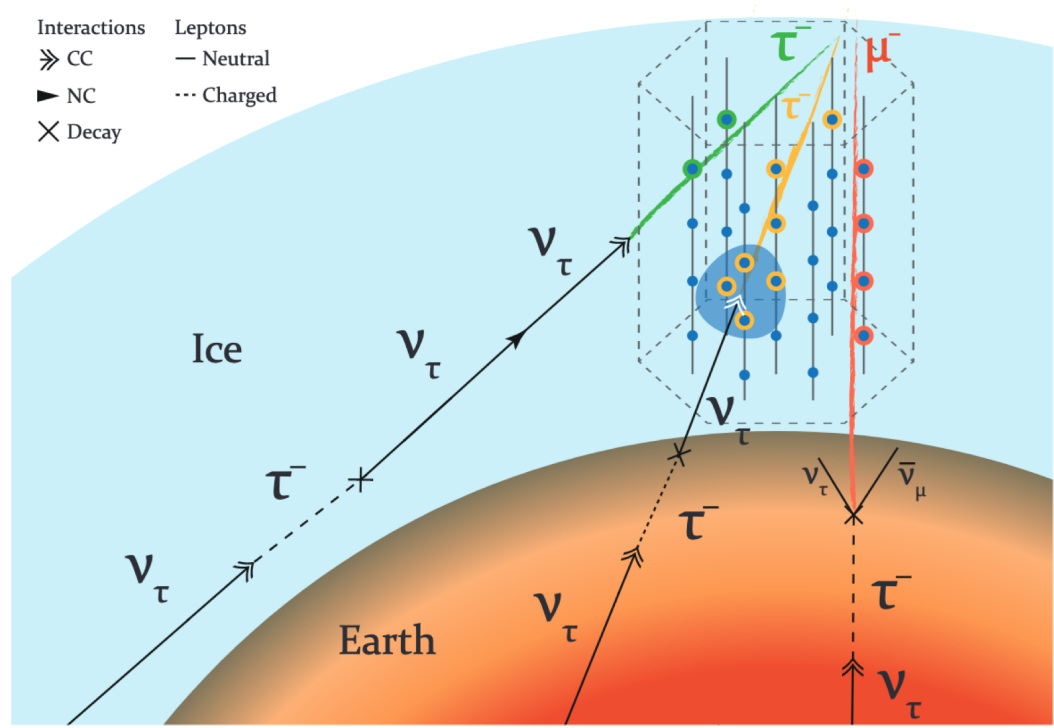

Figure 1: Schematic of neutrino propagation through Earth followed by an event signature in IceCube. EeV tau-neutrino secondaries can be detected in three possible ways, though this work focuses on the rightmost signature. Right: The tau decays before reaching the detector, producing a muon in $\sim 18 \%$ of the cases, which subsequently enters the detector. Center: The interaction vertex is contained in the fiducial volume of the detector in this case, producing a cascade from the charged-current interaction, along with an outgoing tau track. Left: A throughgoing tau track, which is possible for taus at or above $10 \mathrm{PeV}$.

$$
w_{\tau}\left(E_{s h}, E_{\mu}\right)=\int d E_{\tau} \frac{d^{2} \sigma}{d E_{s h} d E_{\tau}}\left(E_{s h}, E_{\tau} ; E_{v}=E_{s h}+E_{\tau}\right) \frac{d N_{\mu}}{d E_{\mu}}\left(E_{\mu} ; E_{\tau}\right) \beta_{\tau \mu}
$$

where $E_{s h}$ is the energy imparted to the hadronic shower in a charge-current interaction, $E_{\mu}$ is the muon energy, $\sigma$ is the neutrino-nucleon cross-section, $d N_{\mu} / d E_{\mu}$ is the energy distribution of muons from tau decay, and $\beta_{\tau \mu}$ is the branching fraction of taus to muons. In this way, specifically by re-weighting simulated muons with the above expression, we can incorporate the contributions of tau neutrinos to throughgoing tracks without the need to generate additional Monte Carlo sets.

In order to estimate sensitivities to UHE neutrinos, our analysis takes into account contributions from atmospheric neutrinos, astrophysical neutrinos described by an unbroken power-law, and an ultra-high energy component. The cosmogenic/UHE astrophysical neutrinos look like an additional contribution to the already measured astrophysical spectrum, but with a modified energy and zenith distribution. Both astrophysical and UHE contributions to track event selections are shown in Fig. 2 in true quantities. We ignore the contribution of atmospheric muons, since the event selection purity is greater than $99.9 \%$ [22]; we also neglect, for the results shown here, the contribution of neutrinos from charmed meson decays as those are sub-leading to the observed astrophysical component. This component will, however, be included in the final analysis. We construct a forward-folding likelihood problem in which we organize the Monte Carlo events in logarithmically spaced bins in reconstructed energy and linearly spaced bins in the cosine of the reconstructed zenith angle.

We then compare the expected distribution in energy and direction to an Asimov set which does not include a UHE component, by means of a modified Poisson likelihood that accounts for 


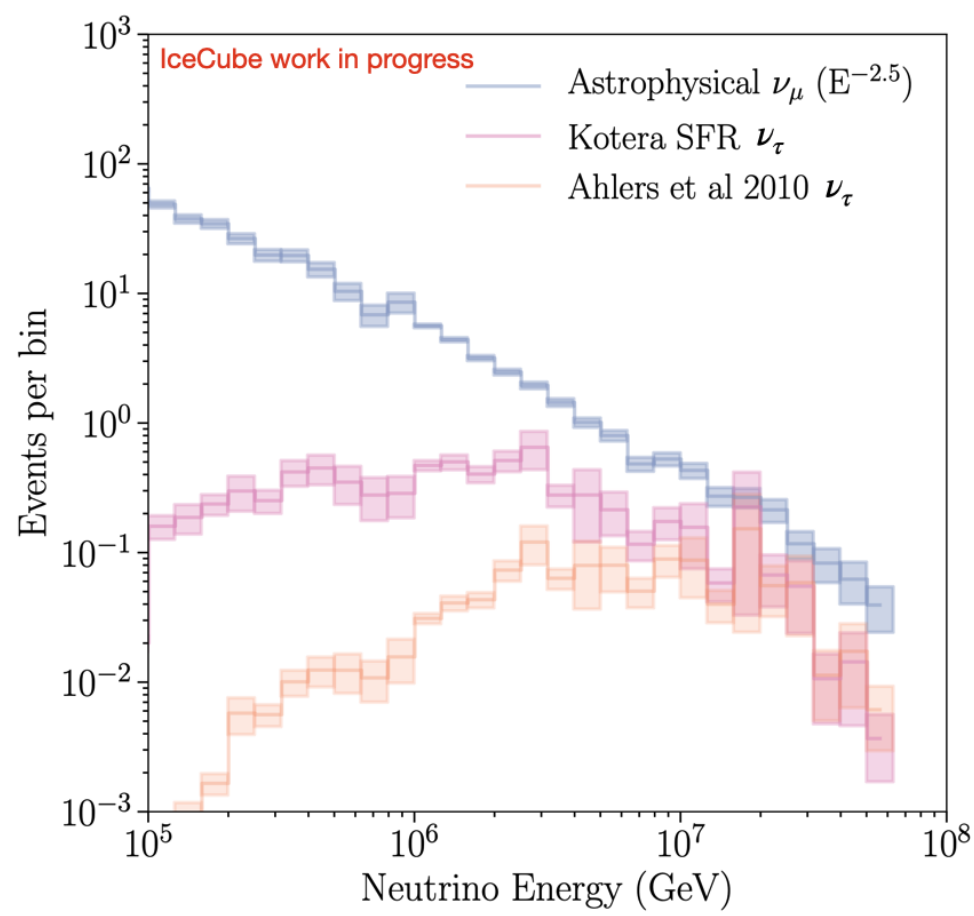

Figure 2: Expected number of muon events in the northern sky, binned in true neutrino energy. This assumes nine years of data and an unbroken power-law with a spectral index of 2.5 for the astrophysical muon-neutrino flux. The GZK contributions according to two models, labeled Kotera and Ahlers et al have comparable rates above a PeV in energy. The error bars encompass statistical monte carlo uncertainties.

Monte Carlo statistical uncertainty. The likelihood function is given by,

$$
\mathcal{L}_{\mathrm{Eff}}(\vec{\theta} \mid k)=\left(\frac{\mu}{\sigma^{2}}\right)^{\frac{\mu^{2}}{\sigma^{2}}+1} \Gamma\left(k+\frac{\mu^{2}}{\sigma^{2}}+1\right)\left[k !\left(1+\frac{\mu}{\sigma^{2}}\right)^{k+\frac{\mu^{2}}{\sigma^{2}}+1} \Gamma\left(\frac{\mu^{2}}{\sigma^{2}}+1\right)\right]^{-1},
$$

where $\vec{\theta}$ is the set of parameters describing the hypothesis being tested, $k$ is the number of observed events in a bin, $\mu \equiv \sum_{i=1}^{m} w_{i}$ is the sum of the weights $w_{i}$ of $m$ Monte Carlo events in a given bin, and similarly $\sigma^{2} \equiv \sum_{i=1}^{m} w_{i}^{2}$. For a detailed explanation and a complete derivation of this function, refer to [24]. The expected event distributions in terms of energy and reconstructed angle quantities are shown in Fig. 3 for nominal choices of the astrophysical flux parameters. Our likelihood problem depends on four variables: the normalization of the conventional atmospheric component, the astrophysical neutrino power-law normalization and spectral index, and the UHE neutrino model normalization. We use the same conventional atmospheric model described in $[22,25]$ and test several benchmark UHE neutrino models given in [26, 27].

\section{Results and conclusions}

We run a Markov Chain Monte Carlo (MCMC) in order to derive allowed regions of parameter space. In our analysis, we use uniform priors on the normalization of each of the components. The astrophysical power-law and the GZK components are given improper uniform priors from 0 

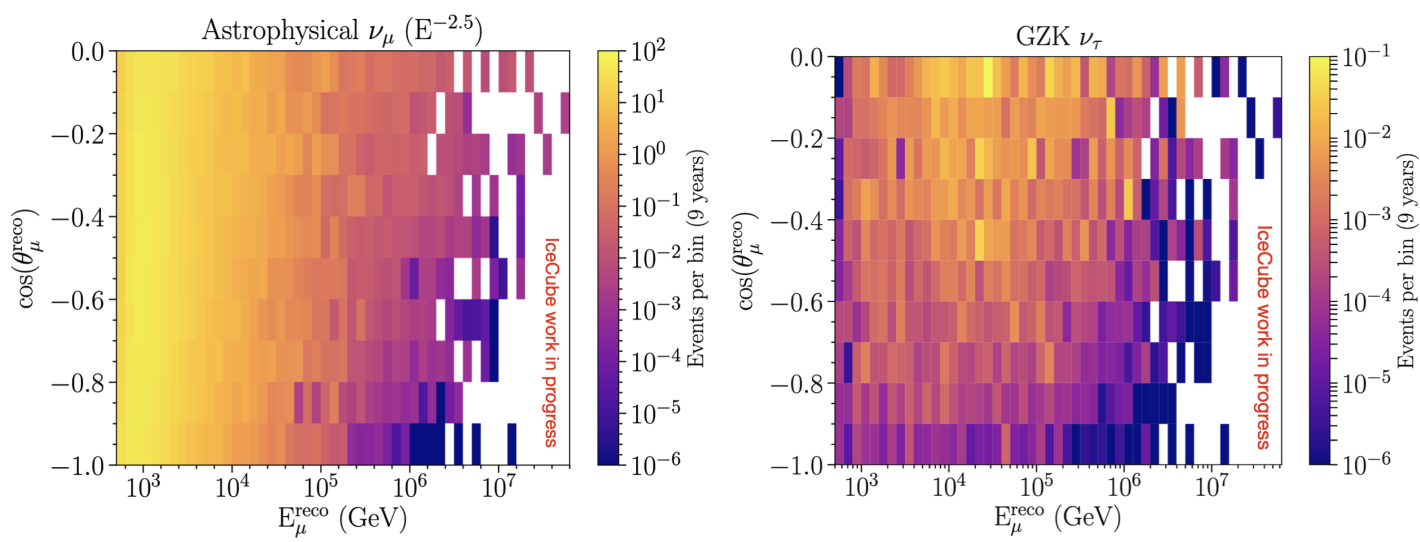

Figure 3: Expected number of events shown in reconstructed quantities for both energy and zenith angle. Left: Muon neutrino events Assuming an equal-flavor astrophysical flux with a spectral index of 2.5 and a normalization of $10^{-18} \mathrm{GeV}^{-1} \mathrm{~cm}^{-2} \mathrm{~s}^{-1} \mathrm{sr}^{-1}$. Right: Muon events in the Northern Sky from GZK tau neutrinos assuming the flux given by [26].

to infinity, while the conventional component is priored by a Gaussian distribution as discussed in [22].

From the MCMC we obtain the model rejection factor, which is defined as the $90 \%$ C.R. (confidence region) upper limit of the chosen model normalization with respect to its nominal prediction. In other words, a rejection factor of 1 or less means the nominal prediction is constrained. We obtain a 90\% C.R UL of 4.11 for the Kotera SFR model and 7 for Ahlers et al. We note that this analysis can be improved by adding the secondary contributions of other neutrino flavors produced in tau decays, which have been shown to contribute equally to events at the detector. Further, the energy reconstruction used in this work provides poor separation of muons above $10 \mathrm{TeV}$, whereas recent work has shown significant improvement in muon energy resolution at high energies, as reported in [28]. As energy separation is crucial to the performance of this analysis, we expect a significant improvement to the sensitivity with updated energy reconstruction. Despite these limitations, the model rejection factors obtained in this work are promising as they are comparable with dedicated analysis upper limits from other experiments. Future work will improve the points noted above and include additional years of data taking.

\section{References}

[1] ICECuBE collaboration, The IceCube high-energy starting event sample: Description and flux characterization with 7.5 years of data, 2011.03545.

[2] ICECUBE collaboration, Characteristics of the diffuse astrophysical electron and tau neutrino flux with six years of IceCube high energy cascade data, Phys. Rev. Lett. 125 (2020) 121104 [2001.09520].

[3] IceCube collaboration, Measurement of the Diffuse Astrophysical Muon-Neutrino Spectrum with Ten Years of IceCube Data, in 36th International Cosmic Ray Conference (ICRC 2019) Madison, Wisconsin, USA, July 24-August 1, 2019, 2019 [1908.09551]. 
[4] IceCube, Fermi-LAT, MAGIC, AGILE, ASAS-SN, HAWC, H.E.S.S., INTEGRAL, Kanata, Kiso, Kapteyn, Liverpool Telescope, Subaru, Swift NuStaR, VERITAS, VLA/17B-403 collaboration, Multimessenger observations of a flaring blazar coincident with high-energy neutrino IceCube-170922A, Science 361 (2018) eaat1378 [1807. 08816].

[5] ICECUBE collaboration, Neutrino emission from the direction of the blazar TXS 0506+056 prior to the IceCube-170922A alert, Science 361 (2018) 147 [1807. 08794].

[6] IceCube collaboration, All Sky Time-Integrated Point Source Searches using 10 years of IceCube Data, PoS ICRC2019 (2020) 851 [1908 . 05993].

[7] C. Righi, A. Palladino, F. Tavecchio and F. Vissani, EeV astrophysical neutrinos from flat spectrum radio quasars, Astron. Astrophys. 642 (2020) A92 [2003.08701].

[8] X. Rodrigues, J. Heinze, A. Palladino, A. van Vliet and W. Winter, Active Galactic Nuclei Jets as the Origin of Ultrahigh-Energy Cosmic Rays and Perspectives for the Detection of Astrophysical Source Neutrinos at EeV Energies, Phys. Rev. Lett. 126 (2021) 191101 [2003.08392].

[9] ICECUBE collaboration, Differential limit on the extremely-high-energy cosmic neutrino flux in the presence of astrophysical background from nine years of IceCube data, Phys. Rev. D 98 (2018) 062003 [1807.01820].

[10] ARA collaboration, Constraints on the diffuse flux of ultrahigh energy neutrinos from four years of Askaryan Radio Array data in two stations, Phys. Rev. D 102 (2020) 043021 [1912.00987].

[11] A. Anker et al., A search for cosmogenic neutrinos with the ARIANNA test bed using 4.5 years of data, JCAP 03 (2020) 053 [1909. 00840].

[12] ANITA collaboration, Constraints on the ultra-high energy cosmic neutrino flux from the fourth flight of ANITA, 1902.04005.

[13] Pierre Auger collaboration, Probing the origin of ultra-high-energy cosmic rays with neutrinos in the EeV energy range using the Pierre Auger Observatory, 1906.07422.

[14] ICECuBE collaboration, The first search for extremely-high energy cosmogenic neutrinos with the IceCube Neutrino Observatory, Phys. Rev. D82 (2010) 072003 [1009 . 1442].

[15] ARIANNA collaboration, A First Search for Cosmogenic Neutrinos with the ARIANNA Hexagonal Radio Array, Astropart. Phys. 70 (2015) 12 [1410.7352].

[16] Pierre Auger collaboration, Improved limit to the diffuse flux of ultrahigh energy neutrinos from the Pierre Auger Observatory, Phys. Rev. D91 (2015) 092008 [1504.05397].

[17] ICECUBE collaboration, Constraints on Ultrahigh-Energy Cosmic-Ray Sources from a Search for Neutrinos above 10 PeV with IceCube, Phys. Rev. Lett. 117 (2016) 241101 [1607. 05886]. 
[18] ARA collaboration, Performance of two Askaryan Radio Array stations and first results in the search for ultrahigh energy neutrinos, Phys. Rev. D93 (2016) 082003 [1507. 08991].

[19] ANITA collaboration, Constraints on the diffuse high-energy neutrino flux from the third flight of ANITA, Phys. Rev. D98 (2018) 022001 [1803.02719].

[20] ICECuBE collaboration, Differential limit on the extremely-high-energy cosmic neutrino flux in the presence of astrophysical background from nine years of IceCube data, Phys. Rev. D98 (2018) 062003 [1807.01820].

[21] I. Safa, A. Pizzuto, C.A. Argüelles, F. Halzen, R. Hussain, A. Kheirandish et al., Observing EeV neutrinos through Earth: GZK and the anomalous ANITA events, JCAP 01 (2020) 012 [1909. 10487].

[22] ICECUBE collaboration, Searching for eV-scale sterile neutrinos with eight years of atmospheric neutrinos at the IceCube Neutrino Telescope, Phys. Rev. D 102 (2020) 052009 [2005.12943].

[23] ICECuBE collaboration, LeptonInjector and LeptonWeighter: A neutrino event generator and weighter for neutrino observatories, Comput. Phys. Commun. 266 (2021) 108018 [2012.10449].

[24] C.A. Argüelles, A. Schneider and T. Yuan, A binned likelihood for stochastic models, JHEP 06 (2019) 030 [1901.04645].

[25] ICECubE collaboration, Measurement of Astrophysical Tau Neutrinos in IceCube's High-Energy Starting Events, 2011.03561.

[26] M. Ahlers, L.A. Anchordoqui, M.C. Gonzalez-Garcia, F. Halzen and S. Sarkar, GZK Neutrinos after the Fermi-LAT Diffuse Photon Flux Measurement, Astropart. Phys. 34 (2010) 106 [1005.2620].

[27] K. Kotera, D. Allard and A.V. Olinto, Cosmogenic Neutrinos: parameter space and detectabilty from PeV to ZeV, JCAP 1010 (2010) 013 [1009.1382].

[28] R. Abbasi et al., A Convolutional Neural Network based Cascade Reconstruction for the IceCube Neutrino Observatory, 2101.11589. 


\section{Full Author List: IceCube Collaboration}

R. Abbasi ${ }^{17}$, M. Ackermann ${ }^{59}$, J. Adams ${ }^{18}$, J. A. Aguilar ${ }^{12}$, M. Ahlers ${ }^{22}$, M. Ahrens ${ }^{50}$, C. Alispach ${ }^{28}$, A. A. Alves Jr. ${ }^{31}$, N. M. $\mathrm{Amin}^{42}$, R. An ${ }^{14}$, K. Andeen ${ }^{40}$, T. Anderson ${ }^{56}$, G. Anton ${ }^{26}$, C. Argüelles ${ }^{14}$, Y. Ashida ${ }^{38}$, S. Axani ${ }^{15}$, X. Bai ${ }^{46}$, A. Balagopal V. ${ }^{38}$, A. Barbano ${ }^{28}$, S. W. Barwick ${ }^{30}$, B. Bastian ${ }^{59}$, V. Basu ${ }^{38}$, S. Baur ${ }^{12}$, R. Bay ${ }^{8}$, J. J. Beatty ${ }^{20,21}$, K.-H. Becker ${ }^{58}$, J. Becker Tjus ${ }^{11}$, C. Bellenghi $^{27}$, S. BenZvi ${ }^{48}$, D. Berley ${ }^{19}$, E. Bernardini ${ }^{59}, 60$, D. Z. Besson ${ }^{34,61}$, G. Binder ${ }^{8,9}$, D. Bindig ${ }^{58}$, E. Blaufuss ${ }^{19}$, S. Blot ${ }^{59}$, M. Boddenberg ${ }^{1}$, F. Bontempo ${ }^{31}$, J. Borowka ${ }^{1}$, S. Böser ${ }^{39}$, O. Botner ${ }^{57}$, J. Böttcher ${ }^{1}$, E. Bourbeau ${ }^{22}$, F. Bradascio ${ }^{59}$, J. Braun ${ }^{38}$, S. Bron $^{28}$, J. Brostean-Kaiser ${ }^{59}$, S. Browne ${ }^{32}$, A. Burgman ${ }^{57}$, R. T. Burley ${ }^{2}$, R. S. Busse ${ }^{41}$, M. A. Campana ${ }^{45}$, E. G. Carnie-Bronca ${ }^{2}$, C. $\mathrm{Chen}^{6}$, D. Chirkin ${ }^{38}$, K. Choi ${ }^{52}$, B. A. Clark ${ }^{24}$, K. Clark ${ }^{33}$, L. Classen ${ }^{41}$, A. Coleman ${ }^{42}$, G. H. Collin ${ }^{15}$, J. M. Conrad ${ }^{15}$, P. Coppin $^{13}$, P. Correa ${ }^{13}$, D. F. Cowen ${ }^{55}, 56$, R. Cross $^{48}$, C. Dappen ${ }^{1}$, P. Dave ${ }^{6}$, C. De Clercq ${ }^{13}$, J. J. DeLaunay ${ }^{56}$, H. Dembinski ${ }^{42}$, K. Deoskar $^{50}$, S. De Ridder ${ }^{29}$, A. Desai ${ }^{38}$, P. Desiati ${ }^{38}$, K. D. de Vries ${ }^{13}$, G. de Wasseige ${ }^{13}$, M. de With ${ }^{10}$, T. DeYoung ${ }^{24}$, S. Dharani ${ }^{1}$, A. Diaz ${ }^{15}$, J. C. Díaz-Vélez ${ }^{38}$, M. Dittmer ${ }^{41}$, H. Dujmovic ${ }^{31}$, M. Dunkman ${ }^{56}$, M. A. DuVernois ${ }^{38}$, E. Dvorak ${ }^{46}$, T. Ehrhardt ${ }^{39}$, P. Eller $^{27}$, R. Engel ${ }^{31,32}$, H. Erpenbeck ${ }^{1}$, J. Evans ${ }^{19}$, P. A. Evenson ${ }^{42}$, K. L. Fan ${ }^{19}$, A. R. Fazely ${ }^{7}$, S. Fiedlschuster ${ }^{26}$, A. T. Fienberg ${ }^{56}$, K. Filimonov ${ }^{8}$, C. Finley ${ }^{50}$, L. Fischer ${ }^{59}$, D. Fox ${ }^{55}$, A. Franckowiak ${ }^{11,59}$, E. Friedman ${ }^{19}$, A. Fritz ${ }^{39}$, P. Fürst ${ }^{1}$, T. K. Gaisser ${ }^{42}$, J. Gallagher $^{37}$, E. Ganster ${ }^{1}$, A. Garcia ${ }^{14}$, S. Garrappa ${ }^{59}$, L. Gerhardt ${ }^{9}$, A. Ghadimi ${ }^{54}$, C. Glaser ${ }^{57}$, T. Glauch ${ }^{27}$, T. Glüsenkamp ${ }^{26}$, A. Goldschmidt $^{9}$, J. G. Gonzalez ${ }^{42}$, S. Goswami ${ }^{54}$, D. Grant ${ }^{24}$, T. Grégoire ${ }^{56}$, S. Griswold ${ }^{48}$, M. Gündüz ${ }^{11}$, C. Günther ${ }^{1}$, C. Haack ${ }^{27}$,

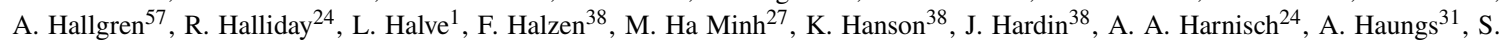
Hauser $^{1}$, D. Hebecker ${ }^{10}$, K. Helbing ${ }^{58}$, F. Henningsen ${ }^{27}$, E. C. Hettinger ${ }^{24}$, S. Hickford ${ }^{58}$, J. Hignight ${ }^{25}$, C. Hill ${ }^{16}$, G. C. Hill ${ }^{2}$, K. D.

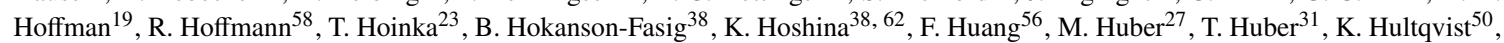
M. Hünnefeld ${ }^{23}$, R. Hussain ${ }^{38}$, S. In ${ }^{52}$, N. Iovine ${ }^{12}$, A. Ishihara ${ }^{16}$, M. Jansson ${ }^{50}$, G. S. Japaridze ${ }^{5}$, M. Jeong ${ }^{52}$, B. J. P. Jones ${ }^{4}$, D. Kang ${ }^{31}$, W. Kang ${ }^{52}$, X. Kang ${ }^{45}$, A. Kappes ${ }^{41}$, D. Kappesser ${ }^{39}$, T. Karg ${ }^{59}$, M. Karl ${ }^{27}$, A. Karle ${ }^{38}$, U. Katz ${ }^{26}$, M. Kauer ${ }^{38}$, M. Kellermann ${ }^{1}$, J. L. Kelley $^{38}$, A. Kheirandish ${ }^{56}$, K. Kin ${ }^{16}$, T. Kintscher ${ }^{59}$, J. Kiryluk ${ }^{51}$, S. R. Klein ${ }^{8,9}$, R. Koirala ${ }^{42}$, H. Kolanoski ${ }^{10}$, T. Kontrimas ${ }^{27}$, L. $\mathrm{Köpke}^{39}$, C. Kopper ${ }^{24}$, S. Kopper ${ }^{54}$, D. J. Koskinen ${ }^{22}$, P. Koundal ${ }^{31}$, M. Kovacevich ${ }^{45}$, M. Kowalski ${ }^{10,59}$, T. Kozynets ${ }^{22}$, E. Kun ${ }^{11}$, N. Kurahashi ${ }^{45}$, N. Lad ${ }^{59}$, C. Lagunas Gualda ${ }^{59}$, J. L. Lanfranchi ${ }^{56}$, M. J. Larson ${ }^{19}$, F. Lauber ${ }^{58}$, J. P. Lazar ${ }^{14,38}$, J. W. Lee ${ }^{52}$, K. Leonard $^{38}$, A. Leszczyńska ${ }^{32}$, Y. Li ${ }^{56}$, M. Lincetto ${ }^{11}$, Q. R. Liu ${ }^{38}$, M. Liubarska ${ }^{25}$, E. Lohfink ${ }^{39}$, C. J. Lozano Mariscal ${ }^{41}$, L. Lu ${ }^{38}$,

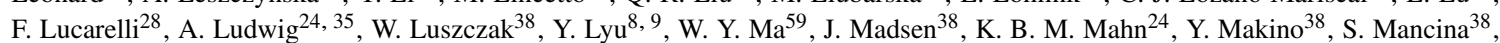

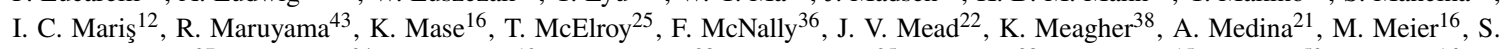
Meighen-Berger $^{27}$, J. Micallef ${ }^{24}$, D. Mockler ${ }^{12}$, T. Montaruli ${ }^{28}$, R. W. Moore ${ }^{25}$, R. Morse ${ }^{38}$, M. Moulai ${ }^{15}$, R. Naab ${ }^{59}$, R. Nagai ${ }^{16}$, U. Naumann ${ }^{58}$, J. Necker ${ }^{59}$, L. V. Nguyễn ${ }^{24}$, H. Niederhausen ${ }^{27}$, M. U. Nisa ${ }^{24}$, S. C. Nowicki ${ }^{24}$, D. R. Nygren ${ }^{9}$, A. Obertacke Pollmann ${ }^{58}$, M. Oehler ${ }^{31}$, A. Olivas ${ }^{19}$, E. O'Sullivan ${ }^{57}$, H. Pandya ${ }^{42}$, D. V. Pankova ${ }^{56}$, N. Park ${ }^{33}$, G. K. Parker ${ }^{4}$, E. N. Paudel ${ }^{42}$, L. Paul ${ }^{40}$, C. Pérez de los $\operatorname{Heros}^{57}$, L. Peters ${ }^{1}$, J. Peterson ${ }^{38}$, S. Philippen ${ }^{1}$, D. Pieloth ${ }^{23}$, S. Pieper ${ }^{58}$, M. Pittermann ${ }^{32}$, A. Pizzuto ${ }^{38}$, M. Plum ${ }^{40}$, Y. Popovych $^{39}$, A. Porcelli ${ }^{29}$, M. Prado Rodriguez ${ }^{38}$, P. B. Price ${ }^{8}$, B. Pries ${ }^{24}$, G. T. Przybylski ${ }^{9}$, C. Raab ${ }^{12}$, A. Raissi ${ }^{18}$, M. Rameez ${ }^{22}$, K. $^{2}$. Rawlins $^{3}$, I. C. Rea ${ }^{27}$, A. Rehman ${ }^{42}$, P. Reichherzer ${ }^{11}$, R. Reimann ${ }^{1}$, G. Renzi ${ }^{12}$, E. Resconi ${ }^{27}$, S. Reusch ${ }^{59}$, W. Rhode ${ }^{23}$, M. Richman $^{45}$, B. Riedel ${ }^{38}$, E. J. Roberts ${ }^{2}$, S. Robertson ${ }^{8,9}$, G. Roellinghoff ${ }^{52}$, M. Rongen ${ }^{39}$, C. Rott ${ }^{49}, 52$, T. Ruhe ${ }^{23}$, D. Ryckbosch ${ }^{29}$, D. Rysewyk $\mathrm{Cantu}^{24}$, I. Safa ${ }^{14,38}$, J. Saffer ${ }^{32}$, S. E. Sanchez Herrera ${ }^{24}$, A. Sandrock ${ }^{23}$, J. Sandroos ${ }^{39}$, M. Santander ${ }^{54}$, S. Sarkar ${ }^{44}$, S. Sarkar ${ }^{25}$, K. Satalecka $^{59}$, M. Scharf ${ }^{1}$, M. Schaufel ${ }^{1}$, H. Schieler ${ }^{31}$, S. Schindler ${ }^{26}$, P. Schlunder ${ }^{23}$, T. Schmidt ${ }^{19}$, A. Schneider ${ }^{38}$, J. Schneider ${ }^{26}$, F. G. Schröder ${ }^{31,42}$, L. Schumacher ${ }^{27}$, G. Schwefer ${ }^{1}$, S. Sclafani ${ }^{45}$, D. Seckel ${ }^{42}$, S. Seunarine ${ }^{47}$, A. Sharma ${ }^{57}$, S. Shefali ${ }^{32}$, M. Silva $^{38}$, B. Skrzypek ${ }^{14}$, B. Smithers ${ }^{4}$, R. Snihur ${ }^{38}$, J. Soedingrekso ${ }^{23}$, D. Soldin ${ }^{42}$, C. Spannfellner ${ }^{27}$, G. M. Spiczak ${ }^{47}$, C. Spiering ${ }^{59,}$, ${ }^{2}$, J. Stachurska $^{59}$, M. Stamatikos ${ }^{21}$, T. Stanev ${ }^{42}$, R. Stein ${ }^{59}$, J. Stettner ${ }^{1}$, A. Steuer ${ }^{39}$, T. Stezelberger ${ }^{9}$, T. Stürwald ${ }^{58}$, T. Stuttard ${ }^{22}$, G. W. Sullivan $^{19}$, I. Taboada ${ }^{6}$, F. Tenholt ${ }^{11}$, S. Ter-Antonyan ${ }^{7}$, S. Tilav ${ }^{42}$, F. Tischbein ${ }^{1}$, K. Tollefson $^{24}$, L. Tomankova ${ }^{11}$, C. Tönnis ${ }^{53}$, S. Toscano $^{12}$, D. Tosi $^{38}$, A. Trettin ${ }^{59}$, M. Tselengidou ${ }^{26}$, C. F. Tung ${ }^{6}$, A. Turcati ${ }^{27}$, R. Turcotte ${ }^{31}$, C. F. Turley ${ }^{56}$, J. P. Twagirayezu ${ }^{24}$, B. $\mathrm{Ty}^{38}$, M. A. Unland Elorrieta ${ }^{41}$, N. Valtonen-Mattila ${ }^{57}$, J. Vandenbroucke ${ }^{38}$, N. van Eijndhoven ${ }^{13}$, D. Vannerom ${ }^{15}$, J. van Santen $^{59}, \mathrm{~S}$.

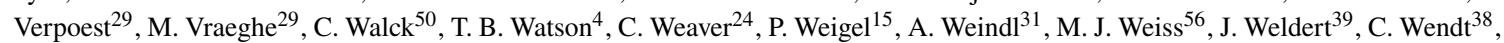
J. Werthebach ${ }^{23}$, M. Weyrauch ${ }^{32}$, N. Whitehorn ${ }^{24,35}$, C. H. Wiebusch ${ }^{1}$, D. R. Williams ${ }^{54}$, M. Wolf ${ }^{27}$, K. Woschnagg ${ }^{8}$, G. Wrede ${ }^{26}$, J.

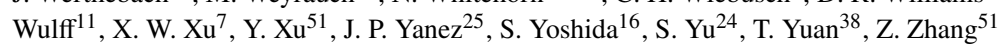

${ }^{1}$ III. Physikalisches Institut, RWTH Aachen University, D-52056 Aachen, Germany

2 Department of Physics, University of Adelaide, Adelaide, 5005, Australia

${ }^{3}$ Dept. of Physics and Astronomy, University of Alaska Anchorage, 3211 Providence Dr., Anchorage, AK 99508, USA

${ }^{4}$ Dept. of Physics, University of Texas at Arlington, 502 Yates St., Science Hall Rm 108, Box 19059, Arlington, TX 76019, USA

5 CTSPS, Clark-Atlanta University, Atlanta, GA 30314, USA

${ }^{6}$ School of Physics and Center for Relativistic Astrophysics, Georgia Institute of Technology, Atlanta, GA 30332, USA

${ }^{7}$ Dept. of Physics, Southern University, Baton Rouge, LA 70813, USA

${ }^{8}$ Dept. of Physics, University of California, Berkeley, CA 94720, USA

${ }^{9}$ Lawrence Berkeley National Laboratory, Berkeley, CA 94720, USA

${ }^{10}$ Institut für Physik, Humboldt-Universität zu Berlin, D-12489 Berlin, Germany

${ }^{11}$ Fakultät für Physik \& Astronomie, Ruhr-Universität Bochum, D-44780 Bochum, Germany

12 Université Libre de Bruxelles, Science Faculty CP230, B-1050 Brussels, Belgium

13 Vrije Universiteit Brussel (VUB), Dienst ELEM, B-1050 Brussels, Belgium

14 Department of Physics and Laboratory for Particle Physics and Cosmology, Harvard University, Cambridge, MA 02138, USA

15 Dept. of Physics, Massachusetts Institute of Technology, Cambridge, MA 02139, USA 
${ }^{16}$ Dept. of Physics and Institute for Global Prominent Research, Chiba University, Chiba 263-8522, Japan

${ }^{17}$ Department of Physics, Loyola University Chicago, Chicago, IL 60660, USA

${ }^{18}$ Dept. of Physics and Astronomy, University of Canterbury, Private Bag 4800, Christchurch, New Zealand

${ }^{19}$ Dept. of Physics, University of Maryland, College Park, MD 20742, USA

${ }^{20}$ Dept. of Astronomy, Ohio State University, Columbus, OH 43210, USA

${ }^{21}$ Dept. of Physics and Center for Cosmology and Astro-Particle Physics, Ohio State University, Columbus, OH 43210, USA

${ }^{22}$ Niels Bohr Institute, University of Copenhagen, DK-2100 Copenhagen, Denmark

${ }^{23}$ Dept. of Physics, TU Dortmund University, D-44221 Dortmund, Germany

${ }^{24}$ Dept. of Physics and Astronomy, Michigan State University, East Lansing, MI 48824, USA

${ }^{25}$ Dept. of Physics, University of Alberta, Edmonton, Alberta, Canada T6G 2E1

${ }^{26}$ Erlangen Centre for Astroparticle Physics, Friedrich-Alexander-Universität Erlangen-Nürnberg, D-91058 Erlangen, Germany

${ }^{27}$ Physik-department, Technische Universität München, D-85748 Garching, Germany

${ }^{28}$ Département de physique nucléaire et corpusculaire, Université de Genève, CH-1211 Genève, Switzerland

${ }^{29}$ Dept. of Physics and Astronomy, University of Gent, B-9000 Gent, Belgium

${ }^{30}$ Dept. of Physics and Astronomy, University of California, Irvine, CA 92697, USA

${ }^{31}$ Karlsruhe Institute of Technology, Institute for Astroparticle Physics, D-76021 Karlsruhe, Germany

${ }^{32}$ Karlsruhe Institute of Technology, Institute of Experimental Particle Physics, D-76021 Karlsruhe, Germany

${ }^{33}$ Dept. of Physics, Engineering Physics, and Astronomy, Queen's University, Kingston, ON K7L 3N6, Canada

${ }^{34}$ Dept. of Physics and Astronomy, University of Kansas, Lawrence, KS 66045, USA

${ }^{35}$ Department of Physics and Astronomy, UCLA, Los Angeles, CA 90095, USA

${ }^{36}$ Department of Physics, Mercer University, Macon, GA 31207-0001, USA

${ }^{37}$ Dept. of Astronomy, University of Wisconsin-Madison, Madison, WI 53706, USA

${ }^{38}$ Dept. of Physics and Wisconsin IceCube Particle Astrophysics Center, University of Wisconsin-Madison, Madison, WI 53706, USA

${ }^{39}$ Institute of Physics, University of Mainz, Staudinger Weg 7, D-55099 Mainz, Germany

${ }^{40}$ Department of Physics, Marquette University, Milwaukee, WI, 53201, USA

${ }^{41}$ Institut für Kernphysik, Westfälische Wilhelms-Universität Münster, D-48149 Münster, Germany

42 Bartol Research Institute and Dept. of Physics and Astronomy, University of Delaware, Newark, DE 19716, USA

${ }^{43}$ Dept. of Physics, Yale University, New Haven, CT 06520, USA

${ }^{44}$ Dept. of Physics, University of Oxford, Parks Road, Oxford OX1 3PU, UK

${ }^{45}$ Dept. of Physics, Drexel University, 3141 Chestnut Street, Philadelphia, PA 19104, USA

${ }^{46}$ Physics Department, South Dakota School of Mines and Technology, Rapid City, SD 57701, USA

${ }^{47}$ Dept. of Physics, University of Wisconsin, River Falls, WI 54022, USA

${ }^{48}$ Dept. of Physics and Astronomy, University of Rochester, Rochester, NY 14627, USA

${ }^{49}$ Department of Physics and Astronomy, University of Utah, Salt Lake City, UT 84112, USA

50 Oskar Klein Centre and Dept. of Physics, Stockholm University, SE-10691 Stockholm, Sweden

${ }^{51}$ Dept. of Physics and Astronomy, Stony Brook University, Stony Brook, NY 11794-3800, USA

52 Dept. of Physics, Sungkyunkwan University, Suwon 16419, Korea

${ }^{53}$ Institute of Basic Science, Sungkyunkwan University, Suwon 16419, Korea

${ }^{54}$ Dept. of Physics and Astronomy, University of Alabama, Tuscaloosa, AL 35487, USA

${ }^{55}$ Dept. of Astronomy and Astrophysics, Pennsylvania State University, University Park, PA 16802, USA

${ }^{56}$ Dept. of Physics, Pennsylvania State University, University Park, PA 16802, USA

${ }^{57}$ Dept. of Physics and Astronomy, Uppsala University, Box 516, S-75120 Uppsala, Sweden

${ }^{58}$ Dept. of Physics, University of Wuppertal, D-42119 Wuppertal, Germany

${ }^{59}$ DESY, D-15738 Zeuthen, Germany

${ }^{60}$ Università di Padova, I-35131 Padova, Italy

${ }^{61}$ National Research Nuclear University, Moscow Engineering Physics Institute (MEPhI), Moscow 115409, Russia

${ }^{62}$ Earthquake Research Institute, University of Tokyo, Bunkyo, Tokyo 113-0032, Japan

\section{Acknowledgements}

USA - U.S. National Science Foundation-Office of Polar Programs, U.S. National Science Foundation-Physics Division, U.S. National Science Foundation-EPSCoR, Wisconsin Alumni Research Foundation, Center for High Throughput Computing (CHTC) at the University of Wisconsin-Madison, Open Science Grid (OSG), Extreme Science and Engineering Discovery Environment (XSEDE), Frontera computing project at the Texas Advanced Computing Center, U.S. Department of Energy-National Energy Research Scientific Computing Center, Particle astrophysics research computing center at the University of Maryland, Institute for Cyber-Enabled Research at Michigan State University, and Astroparticle physics computational facility at Marquette University; Belgium - Funds for Scientific Research (FRS-FNRS and FWO), FWO Odysseus and Big Science programmes, and Belgian Federal Science Policy Office (Belspo); Germany - Bundesministerium für Bildung und Forschung (BMBF), Deutsche Forschungsgemeinschaft (DFG), Helmholtz Alliance for Astroparticle Physics (HAP), Initiative and Networking Fund of the Helmholtz Association, Deutsches Elektronen Synchrotron (DESY), and High Performance Computing cluster of the RWTH Aachen; Sweden - Swedish Research Council, Swedish Polar Research Secretariat, Swedish National Infrastructure for Computing (SNIC), and Knut and Alice Wallenberg Foundation; Australia - Australian 
Research Council; Canada - Natural Sciences and Engineering Research Council of Canada, Calcul Québec, Compute Ontario, Canada Foundation for Innovation, WestGrid, and Compute Canada; Denmark - Villum Fonden and Carlsberg Foundation; New Zealand Marsden Fund; Japan - Japan Society for Promotion of Science (JSPS) and Institute for Global Prominent Research (IGPR) of Chiba University; Korea - National Research Foundation of Korea (NRF); Switzerland - Swiss National Science Foundation (SNSF); United Kingdom - Department of Physics, University of Oxford. 